\title{
Pragmatic Recommendations for Infection Prevention and Control Practices for Healthcare Facilities in Low- and Middle-Income Countries during the COVID-19 Pandemic
}

\author{
Natalie Cobb, ${ }^{1 *}$ Alfred Papali, ${ }^{2}$ Luigi Pisani, ${ }^{3,4}$ Marcus J. Schultz, ${ }^{4,5,6}$ and Juliana C. Ferreira ${ }^{7}$ for the COVID-LMIC Task Force and \\ the Mahidol-Oxford Research Unit (MORU) \\ ${ }^{1}$ Division of Pulmonary, Critical Care, and Sleep Medicine, University of Washington, Seattle, Washington; ${ }^{2}$ Division of Pulmonary and Critical Care \\ Medicine, Atrium Health, Charlotte, North Carolina; ${ }^{3}$ Section of Operative Research, Doctors with Africa, CUAMM, Padova, Italy; ${ }^{4}$ Mahidol-Oxford \\ Tropical Medicine Research Unit (MORU), Mahidol University, Bangkok, Thailand; ${ }^{5}$ Department of Intensive Care, Laboratory of Experimental \\ Intensive Care and Anesthesiology (L.E.I.C.A), Amsterdam University Medical Centers, Amsterdam, The Netherlands; ${ }^{6}$ Nuffield Department of \\ Medicine, Oxford University, Oxford, United Kingdom; ${ }^{7}$ Divisao de Pneumologia, Instituto do Coracao, Hospital das Clinicas HCFMUSP,
} Faculdade de Medicina da Universidade de Sao Paulo, São Paulo, Brazil

\begin{abstract}
Infection prevention and control (IPC) strategies are key in preventing nosocomial transmission of COVID-19. Several commonly used IPC practices are resource-intensive and may be challenging to implement in resource-constrained settings. An international group of healthcare professionals from or with experience in low- and middle-income countries (LMICs) searched the literature for relevant evidence. We report on a set of pragmatic recommendations for hospital-based IPC practices in resource-constrained settings of LMICs. For cases of confirmed or suspected COVID-19, we suggest that patients be placed in a single isolation room, whenever possible. When single isolation rooms are unavailable or limited, we recommend cohorting patients with COVID-19 on dedicated wards or in dedicated hospitals. We also recommend that cases of suspected COVID-19 be cohorted separately from those with confirmed disease, whenever possible, to minimize the risk of patient-to-patient transmission in settings where confirmatory testing may be limited. We suggest that healthcare workers be designated to care exclusively for patients with COVID-19, whenever possible, as another approach to minimize nosocomial spread. This approach may also be beneficial in conserving limited supplies of reusable personal protective equipment (PPE). We recommend that visitors be restricted for patients with COVID-19. In settings where family members or visitors are necessary for caregiving, we recommend that the appropriate PPE be used by visitors. We also recommend that education regarding hand hygiene and donning/doffing procedures for PPE be provided. Last, we suggest that all visitors be screened for symptoms before visitation and that visitor logs be maintained.
\end{abstract}

\section{INTRODUCTION}

Hospital-related transmission of COVID-19 among patients and healthcare providers has been reported. ${ }^{1-4}$ As COVID-19 is a highly contagious disease, ${ }^{5}$ strategies to mitigate the spread of COVID-19 within healthcare settings are crucial. Studies in the United States and other high-income countries (HICs) have demonstrated that the risk of hospital-associated transmission is low when strict infection control measures are applied. ${ }^{6,7}$ However, many commonly used infection prevention and control (IPC) strategies are resource-intensive, including the use of single-occupancy hospital isolation rooms and single-use personal protective equipment (PPE). In resource-constrained settings, limited availability of these resources and limited staffing may pose challenges because COVID-19 cases continue to increase. Recommendations to minimize the risk of nosocomial spread in resourceconstrained settings in low- and middle-income countries (LMICs) are needed. We report on a set of pragmatic recommendations for IPC practices of healthcare facilities in LMICs focused on three key areas: 1) isolation and cohorting of patients, 2) cohorting of healthcare workers (HCWs), and 3) guidance regarding hospital visitors (Table 1).

\section{METHODS}

A full description of the methods is provided in the Appendix. In short, we formulated a set of clearly defined questions

\footnotetext{
*Address correspondence to Natalie Cobb, Division of Pulmonary, Critical Care, and Sleep Medicine, University of Washington Medical Center, 1959 NE Pacific St., Box 356522, Seattle, WA 98195. E-mail: ncobb@uw.edu
}

regarding IPC practices for healthcare facilities caring for COVID-19 patients in LMICs. These questions were reviewed for content and clarity by other members of the COVID-LMIC Task Force. After approval, members of the subgroup were assigned to search the literature for evidence to answer each question.

The literature search was performed in PubMed and the Cochrane Library. References of selected articles were also reviewed to identify relevant publications. Furthermore, we reviewed existing guidelines from the WHO, Africa CDC, U.S. CDC, and European CDC (ECDC).

We selected relevant publications, appraised the evidence, and classified the quality of evidence as high, moderate, low, or very low. Recommendations were rated as strong or weak, depending on the quality of evidence and several other factors such as availability, affordability, and feasibility in LMICs. A strong recommendation was worded as "we recommend. .." and a weak recommendation as "we suggest. ..," followed by the quality of evidence. A number of recommendations could remain "ungraded" (UG), when, in the opinion of the subgroup members, such recommendations were not conducive for the process described previously (Appendix Table A2). The recommendations were reviewed by the subgroup in an iterative process and were later reviewed by the entire Task Force in two rounds.

\section{QUESTIONS}

We formulated three clearly defined questions regarding IPC practices in healthcare facilities:

1. Should hospitalized patients with suspected or confirmed COVID-19 in LMICs be cohorted and, if so, how? 
2. Should HCWs taking care of hospitalized COVID-19 patients in LMICs care exclusively for those patients?

3. Should visiting policies be altered for patients hospitalized with COVID-19 in LMICs?

Should hospitalized patients with suspected or confirmed COVID-19 in LMICs be cohorted and, if so, how? Rationale. Infection prevention and control strategies are an important part of limiting transmission of respiratory viruses in healthcare settings. A key aspect of IPC involves early detection and isolation of cases. In HICs, isolation within healthcare facilities is often achieved by using single-occupancy isolation rooms. In LMICs, this approach may not always be feasible because of limited availability of isolation rooms. A 2020 Cochrane review found that lack of adequate isolation rooms is perceived as major barrier to IPC practices by HCWs, particularly in LMICs. ${ }^{8}$ When isolation rooms are limited, are cohorting strategies effective in preventing nosocomial transmission? What cohorting strategies should be used to isolate patients with COVID-19 or suspected COVID-19?

Search results. We searched PubMed and the Cochrane Library using combinations of the following Medical Subject Headings (MeSH) or free-text words: "cohorting AND COVID19," "patient isolation AND COVID-19," "infection control/ methods AND COVID-19," "respiratory tract infections/ prevention and control AND COVID-19," and "pandemics/ prevention and control AND COVID-19" through November 1, 2020. The PubMed search yielded 7,824 articles, including 89 systematic reviews. Overall, 2,066 articles were from LMICs. Few studies from LMICs directly addressed the question of interest. As such, we discuss the literature and guidelines from both HICs and LMICs, which may inform recommendations. One Cochrane systematic review addressing IPC practices to prevent transmission of respiratory viruses was identified.

Evidence. Evidence regarding the efficacy of cohorting patients with suspected or confirmed COVID-19 in reducing the risk of nosocomial transmission is limited. However, cohorting strategies have previously been studied for other respiratory viruses, such as respiratory syncytial virus and SARS coronavirus. A 2011 Cochrane review evaluated the use of various physical interventions to minimize the transmission of respiratory viruses. ${ }^{9}$ In this review, four studies were identified that reported cohorting as an effective measure in reducing virus spread. ${ }^{9}$ One controlled before and after study showed a reduction in nosocomial transmission of respiratory syncytial virus using cohorting with a decrease from 5.33 to 1.23 infections per 1,000/patient days of care. ${ }^{10}$ Experience from the 2003 SARS outbreak in Singapore also suggests that cohorting on an open ward may be effective in an outbreak setting. ${ }^{11}$ In this observational study, 70 patients exposed to SARS were cohorted for monitoring. Patients who developed symptoms concerning SARS infection were removed from the cohort and isolated. The authors reported that within the cohort, there was no observed secondary transmission. ${ }^{11}$

A similar approach has been used in Singapore during the COVID-19 pandemic with the creation of "respiratory surveillance wards," which were designated for any patients with respiratory symptoms. ${ }^{12}$ When cases of SARS-CoV-2 were confirmed, patients were moved to a separate isolation ward. Among staff and patients exposed to SARS-CoV-2, only one patient developed COVID-19 after exposure. Another strategy described for COVID-19 in the United Kingdom relies on the use of a triage tool to assign patients to single-room isolation versus cohorting based on the likelihood of COVID-19 infection and the risk of a poor outcome. ${ }^{13}$ In a study of 93 patients that describes this approach, no cases of symptomatic hospital-acquired infections were found among cohorted patients. ${ }^{13}$ Few studies from LMICs have been published discussing approaches and challenges to cohorting patients with confirmed or suspected COVID-19. ${ }^{14,15}$

Guidelines from the WHO, CDC, and ECDC recommend that patients with COVID-19 be placed in single isolation rooms, when possible. ${ }^{3,16-19}$ When it is not feasible to isolate cases in single rooms, the WHO recommends cohorting patients according to their status as confirmed, probable, or suspected COVID-19; probable cases are defined as "suspect cases for whom testing is inconclusive or not available." ${ }^{18-20}$

TABLE 1

Recommendations for COVID-19-related infection prevention and control practices for healthcare facilities in LMICs

\begin{tabular}{|c|c|c|}
\hline 1 & Isolation and cohorting & $\begin{array}{l}\text { In LMICs, when cases of COVID-19 are sporadic, we suggest that persons with suspected or } \\
\text { confirmed COVID-19 be placed in single isolation rooms, if available (weak recommendation, } \\
\text { very low quality of evidence). }\end{array}$ \\
\hline 2 & Isolation and cohorting & $\begin{array}{l}\text { When there are no single isolation rooms or when there is a surge of cases, we recommend that } \\
\text { patients with confirmed COVID-19 be cohorted together (strong recommendation, low quality of } \\
\text { evidence). In addition, we recommend that patients with suspected disease be cohorted } \\
\text { separately or placed in isolation rooms (strong recommendation, low quality of evidence). }\end{array}$ \\
\hline 3 & Healthcare workers & $\begin{array}{l}\text { In LMICs, when possible, we suggest that teams of dedicated healthcare professionals be } \\
\text { designated to care exclusively for COVID-19 patients (weak recommendation, very low quality of } \\
\text { evidence). }\end{array}$ \\
\hline 4 & Healthcare workers & $\begin{array}{l}\text { When it is not possible to have dedicated teams, we recommend strict adherence to local PPE } \\
\text { guidelines and hand hygiene to minimize the risk of cross-contamination or transmission } \\
\text { between healthcare personnel and patients (strong recommendation, low quality of evidence). }\end{array}$ \\
\hline 5 & Visitors & $\begin{array}{l}\text { For hospitals in LMICs where staff can provide all care, we suggest that visitor restrictions be } \\
\text { placed in accordance with local regulations and guidelines (ungraded). }\end{array}$ \\
\hline 6 & Visitors & $\begin{array}{l}\text { When visitors are necessary for the care of patients, we recommend keeping the number of visitors } \\
\text { to a minimum (strong recommendation, very low quality of evidence). We also recommend that } \\
\text { visitors use appropriate PPE and that education regarding hand hygiene, and donning and } \\
\text { doffing of PPE be provided (strong recommendation, very low quality of evidence). }\end{array}$ \\
\hline 7 & Visitors & $\begin{array}{l}\text { We suggest that banners be placed at the entrance of healthcare facilities to inform visitors about } \\
\text { symptoms of COVID-19 and that visitors be screened for symptoms before visiting. Visitor logs } \\
\text { should also be maintained (weak recommendation, very low quality of evidence). }\end{array}$ \\
\hline
\end{tabular}


Similarly, the ECDC guidelines recommend placing confirmed COVID-19 cases in a separate ward or area of the hospital. ${ }^{3}$ For settings in which a limited number of isolation rooms are available, one suggestion has been that patients with confirmed COVID-19 should be cohorted together and that isolation rooms should be reserved for patients with suspected disease. $^{21}$ The Africa CDC recommends preferentially admitting suspected cases to single rooms; however, when this is not feasible, suspected cases may be admitted to dedicated wards, separate from confirmed COVID-19 cases. ${ }^{22}$ It is also recommended that confirmed cases be placed in bays according to mild, moderate, severe, and critical disease severity. ${ }^{22}$

When using a cohorting approach, there are additional precautions that must be taken into consideration, including physical distancing and ward ventilation. The recommendations regarding physical distance between patients on an open ward vary with a minimum suggested distance of $1-2 \mathrm{~m} .{ }^{17}$ The Severe Acute Respiratory Infections Treatment Center manual from the WHO advise that beds be placed at least $2 \mathrm{~m}$ apart for persons with suspected COVID-19. ${ }^{20}$ Adequate ventilation is also an important consideration and further discussed in the "Safety" section of this series. ${ }^{23}$ Guidelines from the WHO, CDC, and Africa CDC additionally recommend the use of engineering controls, such as glass or plastic barriers between staff and patients in reception, screening, and triage areas ${ }^{20,24}$ and the use of curtains or other physical barriers in shared patient areas. ${ }^{16}$

Availability, feasibility, affordability, and safety. Single isolation rooms may be ideal for patients with suspected or confirmed COVID-19, when available. However, this approach is likely impractical in many LMIC settings, given the limited availability of isolation rooms, particularly during surges of COVID-19. Cohorting patients with COVID-19 on dedicated wards according to confirmed or suspected infection is likely a more feasible approach. As local strategies are devised, they may take into consideration the availability of any singleoccupancy isolation rooms. If no single isolation rooms are available, ward cohorting strategies based on disease status (confirmed, probable, and suspected) are likely safe and affordable.

Recommendations and suggestions.

1. In LMICs, when cases of COVID-19 are sporadic, we suggest that persons with suspected or confirmed COVID-19 be placed in single isolation rooms, if available (weak recommendation, very low quality of evidence).

2. In LMICs, when there are no single isolation rooms, or when there is a surge of cases, we recommend that patients with confirmed COVID-19 be cohorted together (strong recommendation, low quality of evidence). In addition, we recommend that patients with suspected disease be cohorted separately or placed in isolation rooms (strong recommendation, low quality of evidence).

Should HCWs taking care of hospitalized COVID-19 patients in LMICs care exclusively for those patients? Rationale. Asymptomatic infection and transmission of SARSCoV-2 has been well described. ${ }^{25}$ Given the increased risk of COVID-19 among HCWs, ${ }^{26}$ one concern regarding HCWs caring for both COVID-19 and non-COVID-19 patients is that they may inadvertently spread the disease. Creating dedicated teams of HCWs for patients with COVID-19 has several potential advantages, including rational use of non-disposable
PPE, fewer healthcare professionals exposed to COVID-19, and a lower risk of HCWs inadvertently transmitting COVID-19 to patients. However, limitations of this approach may include shortages of healthcare personnel, particularly during surges, and an increased risk of burnout syndrome among HCWs. ${ }^{27}$

Search results. We searched PubMed using combinations of the following MeSH terms and free-text words: "COVID-19 AND health personnel," "coronavirus AND health personnel," "Middle East Respiratory Syndrome (MERS) Coronavirus AND health personnel," and "SARS virus AND health personnel" through November 1, 2020. The search yielded 2,980 studies, including 44 systematic reviews. Among them, 765 studies were from LMICs; no studies from LMICs that directly addressed the question of interest were identified. We discuss studies and guidelines related to HCWs caring for patients with COVID-19, SARS, and MERS, from both HICs and LMICs, which can inform recommendations.

Evidence. Several studies have shown an increased risk of COVID-19 infection among HCWs. ${ }^{28}$ Moreover, reports of nosocomial transmission of COVID-19 have documented transmission among HCWs as well as nonclinical staff, patients, and caregivers. ${ }^{1,4,29}$ A recent report of an outbreak in Hanoi, Vietnam, during March-April found that $91.3 \%$ of confirmed COVID-19 cases detected had a history of admission to a hospital or worked in or visited a hospital complex. ${ }^{4}$ Another study from Ghana found a high rate of COVID-19 among staff at a primary care clinic. ${ }^{30}$ Although nosocomial transmission has been documented, no studies were found that evaluated the role of cohorting $\mathrm{HCW}$ s in reducing the risk of COVID-19 transmission.

There are several reports during the SARS and MERS outbreaks in which entire hospitals or separate hospital wards were designated for the care of SARS or MERS patients. During the 2003 SARS outbreak in Taiwan, the government implemented dedicated SARS hospitals across the country as part of the strategy to deal with the surge of patients. ${ }^{31} \mathrm{~A}$ similar approach was considered in Toronto, Canada, however, implementing dedicated SARS hospitals proved challenging and instead many hospitals were prepared to care for SARS patients. ${ }^{32}$ When the second wave occurred in Toronto, four hospitals became designated SARS hospitals. ${ }^{32}$ In some settings, these strategies also resulted in dedicated teams of healthcare professionals, including physicians, nurses, and allied healthcare professions. ${ }^{33}$ However, in one report from Canada, HCWs who were employed in more than one hospital transmitted SARS between institutions, demonstrating the risk of transmission from HCWs. ${ }^{34}$

Evidence from the Ebola virus disease (EVD) in West Africa demonstrated that healthcare delivery settings play major roles in the propagation of EVD outbreaks, ${ }^{35}$ but most HCW infections were associated with general healthcare and home settings and not with dedicated EVD settings. ${ }^{36}$ This may also prove true for SARS-CoV-2, although several nosocomial outbreaks of SARS-CoV-2 have been described as well. ${ }^{4,37}$ Interestingly, during the EVD outbreaks in Sierra Leone, although many of the infected HCWs in the study were aware of the time and incident that resulted in exposure, they delayed presentation to treatment facilities for the fear of stigma associated with EVD infection. ${ }^{36}$ The issue of stigma associated with COVID-19 disease is a common topic among populations and HCWs in LMICs. Several strategies have been proposed to protect HCWs and to mitigate stigma. ${ }^{38,39}$ 
The WHO IPC guidelines recommend that a team of HCWs should be designated to care exclusively for suspected or confirmed cases of COVID-19, where possible. ${ }^{18}$ Similarly, the WHO manual for severe acute respiratory infections treatment centers recommends "where possible, cohort HCWs to care exclusively for people with COVID-19 to reduce the risk of transmission due to inadvertent infection control breaches."20 The CDC also suggests that facilities should consider designating entire units to care for COVID-19 patients, with dedicated healthcare professionals, as a measure to limit healthcare professionals' exposure and conserve PPE. ${ }^{16}$

In settings where PPE is limited or in which it must be reused, cohorting of HCWs may be particularly relevant in preserving supplies. However, it is important to recognize the risk posed to HCWs. Adequacy of PPE and suboptimal handwashing have been identified as important risk factors for infection. ${ }^{26,40}$ These findings underscore the importance of ensuring that HCWs caring for patients with COVID-19 are equipped with PPE and trained in donning, doffing, and hand hygiene practices.

Given the increased risk of infection among $\mathrm{HCW}$ caring for COVID-19 patients, it may be appropriate to consider additional precautions in certain settings. For instance, the Africa CDC recommends that staff with underlying medical conditions, such as HIV, be allowed to care for non-COVID-19 patients. ${ }^{24}$ These guidelines also recommend that HCWs should not have direct contact with family members while working at isolation centers and should quarantine before reuniting with family. ${ }^{24}$ Last, HCWs caring for COVID-19 patients have reported high rates of depression, anxiety, or distress, ${ }^{27}$ and providing adequate support to $\mathrm{HCW}$ s will be key for mitigating potential adverse effects on mental health.

Availability, feasibility, affordability, and safety. The implementation of dedicated teams of HCWs to care for COVID-19 patients in LMICs may decrease the risk of nosocomial transmission and improve safety for both patients and healthcare providers. Having a dedicated COVID-19 team of healthcare professionals is practical and should not result in extra costs if teams can be organized without having to hire additional staff. However, this approach may be impractical during extreme shortages of personnel in resourceconstrained settings.

Recommendations and suggestions.

1. In LMICs, when possible, we suggest that teams of dedicated healthcare professionals be designated to care exclusively for COVID-19 patients (weak recommendation, very low quality of evidence).

2. When it is not possible to have dedicated teams, we recommend strict adherence to local PPE guidelines and hand hygiene to minimize the risk of cross-contamination or transmission between healthcare personnel and patients (strong recommendation, low quality of evidence).

Should visiting policies be altered for hospitalized patients with COVID-19 in LMICs? Rationale. In healthcare facilities, visitation of patients with COVID-19 has been limited in many HICs to minimize the risk of healthcare-associated transmission. However, in LMIC settings, family members are sometimes involved in providing essential care, such as bathing, feeding, and turning patients. It is unclear whether visitors should be restricted in these settings. If visitors are allowed, what policies should be implemented to protect patients, healthcare providers, and visitors?

Search results. We searched PubMed using combinations of the following MeSH terms and free-text words: "COVID-19 AND visitors to patients," "coronavirus AND visitors to patients," "MERS Coronavirus AND visitors to patients," and "SARS virus AND visitors to patients" through November 1 , 2020. The PubMed search yielded 48 studies; seven studies were from LMICs. No studies were identified from LMICs that directly addressed the question of interest. We discuss studies and guidelines related to visitors for patients with COVID-19, SARS, and MERS, from both HICs and LMICs, which can inform recommendations.

Evidence. There are several concerns around hospital visitation in the setting of the COVID-19 pandemic. Given that transmission of COVID-19 during the asymptomatic or presymptomatic period has been described, ${ }^{41-43}$ a major concern is that hospital visitors may transmit the virus to patients and healthcare professionals, risking hospital outbreaks such as those reported for SARS. ${ }^{32,44}$ There is also risk of transmission from patients to visitors and, subsequently, risk of visitor transmission to the community. From the SARS and MERS epidemics, there is a large body of literature documenting transmission among hospital visitors. ${ }^{45-56}$ Early in the COVID-19 outbreak in Wuhan, China, one report found that $10 \%$ of COVID-19 cases were related to hospital visits. ${ }^{57}$

The use of visitor restrictions during the COVID-19 pandemic has included a range of policies from no-visitor policies to restricting the number of visitors paired with visitor screening. A survey of intensive care units in the United States found that nearly all hospitals implemented "no-visitor" policies, with certain exceptions at the end-of-life. ${ }^{58}$ Strict visitation policies were also implemented by the National Health Service in the United Kingdom. ${ }^{59}$ In Taiwan, most hospitals changed visiting policies, with some implementing no-visitor policies and others restricting the number of visitors and visiting hours, paired with symptom and temperature monitoring to screen visitors. ${ }^{60}$ In hospices and nursing homes, which have been significantly affected by the COVID-19 pandemic, restrictions to visitation have been put in place in many countries, including Taiwan and the United States. ${ }^{61,62}$

Although evidence regarding the efficacy of these approaches for COVID-19 is limited, policies are derived from experiences during other respiratory viral epidemics. Infection control strategies including restricted visitation, screening visitors for symptoms, and maintaining visitor logs have been described previously. ${ }^{63,64}$ These were part of infection control measures that were effective in halting nosocomial outbreaks of MERS, SARS, and influenza H1N1. ${ }^{46,52,65-73}$ In Canada during the 2003 SARS epidemic, there was a Ministry of Health ban on all nonessential staff members and visitors, except on compassionate grounds (e.g., a parent visiting an ill child). ${ }^{34}$ Visitor logs were used when visitors were allowed to facilitate contact tracing if a transmission occurred..$^{34}$ In other settings, visitors were restricted but not banned as part of infection control strategies used during the SARS and MERS epidemics. In those cases, visitors were screened for symptoms and required to wear PPE during visits. ${ }^{31,33,72,74-76}$ Required PPE ranged from complete contact and droplet precautions (gown, gloves, and N95 respirators) to simply a surgical mask. ${ }^{33,52,72}$ 
The CDC, Africa CDC, and WHO have issued recommendations regarding visitation in the context of the COVID-19 pandemic. The recommendations issued by the CDC for nonAmerican facilities include restricting visitors, using visual alerts about symptoms, and screening for symptoms. The CDC recommends that during periods of widespread community transmission of COVID-19, visitor access to healthcare facilities should be restricted. Only visitors essential for helping to provide patient care and/or caring for pediatric patients should be allowed. If visitors are allowed, they should be instructed about hand hygiene, cough etiquette, and mask use. $^{77}$ The WHO recommends that visitors be reduced to a minimum and that visitors follow guidelines regarding hand hygiene as well as contact and droplet precautions before entering the room of a suspected or confirmed COVID-19 patient. A visitor log is also recommended. ${ }^{18}$ The Africa CDC outlines two strategies for caregivers: 1) restricting all caregivers and recruiting additional healthcare aides, or 2) allowing caregivers with restrictions. ${ }^{78}$ When caregivers are allowed, it is recommended that the number be limited to one per patient and that caregivers receive practical instruction in infection prevention practices. ${ }^{78}$

In resource-constrained settings in LMICs where caregivers are allowed, one challenge may be ensuring adequate availability of PPE for use by visitors. In addition, ensuring adequate instruction and supervision of caregivers regarding hand hygiene, proper use of PPE, and attention to surface contamination may also pose a challenge. In a study of 228 health facilities in Tanzania, compliance with hand hygiene and disinfection practices was low among HCWs. ${ }^{79}$ Poor compliance among HCWs could result in inadequate visitor instruction and increase the risk of healthcare-associated transmission.

The ethics of banning or restricting visitors are challenging, and policies must balance the importance of public health and patient-centered care. In instances where visitor bans are implemented, it is important to consider circumstances that may require exceptions and alternative approaches to supporting families and patients. ${ }^{80}$ Exceptions to visitor restrictions may need to be considered under compassionate circumstances, such as near the end-of-life. Identifying ways that spiritual support may be provided for patients and families when requested is also important; one hospital in the United States describes facilitating "visits" during which spiritual leaders or clergy may pray outside of patient rooms or with family members. ${ }^{81}$ When visitation is restricted, systems must also be developed for family communication. In Italy and South Africa, remote family visits through video calls and virtual family conferences have been used. ${ }^{82,83}$ During the SARS epidemic in Hong Kong, helpdesks for relatives were established to provide emotional and communication support. ${ }^{84}$

Availability, affordability, safety, and feasibility. Implementing visitor restrictions and symptom screening is likely feasible in many settings and may enhance safety for patients, healthcare personnel, and the community. In settings where visitors are allowed, visitor logs may also facilitate contact tracing in the event of an outbreak. Similarly, the use of visual alerts in the form of signs or banners at the entrance of healthcare facilities is low cost and could be easily implemented. If visitors need to be actively involved in the care of patients with COVID-19 because of a lack of human resources, adequate PPE and training for visitors should be ensured. Adequate availability of PPE, such as gowns, gloves, and surgical or N95 masks, may be a considerable barrier in resource-constrained settings.

Recommendations and suggestions.

1. For hospitals in LMICs where staff can provide all care, we suggest that visitor restrictions be placed in accordance with local regulations and guidelines (UG statement).

2. When visitors are necessary for the care of patients, we recommend keeping the number of visitors to a minimum (strong recommendation, very low quality of evidence). We also recommend that visitors use appropriate PPE and that education regarding hand hygiene, and donning and doffing of PPE be provided (strong recommendation, very low quality of evidence).

3. We suggest that banners be placed at the entrance of healthcare facilities to inform visitors about symptoms of COVID-19 and that visitors be screened for symptoms before visiting. Visitor logs should also be maintained (weak recommendation, very low quality of evidence).

Received August 12, 2020. Accepted for publication December 20, 2020.

Published online January 6, 2021.

Note: The Appendix can be found at www.ajtmh.org.

Financial support: Open access fees for this article and all others in this issue were supported by the Wellcome Trust of Great Britain.

Authors' addresses: Natalie Cobb, University of Washington, Seattle, WA, E-mail: ncobb@uw.edu. Alfred Papali, Atrium Health, Charlotte, NC, E-mail: alfred.papali@atriumhealth.org. Luigi Pisani, Doctors with Africa, CUAMM, Padova, Italy, and Mahidol-Oxford Tropical Medicine Research Unit (MORU), Mahidol University, Bangkok, Thailand, E-mail: luigipisani@gmail.com. Marcus J. Schultz, Mahidol University, Bangkok, Thailand, and Amsterdam University Medical Center, Amsterdam, The Netherlands, E-mail: marcus.j.schultz@gmail.com. Juliana C. Ferreira, University of São Paulo, São Paulo, Brazil, E-mail: juliana.ferreira@hc.fm.usp.br.

This is an open-access article distributed under the terms of the Creative Commons Attribution (CC-BY) License, which permits unrestricted use, distribution, and reproduction in any medium, provided the original author and source are credited.

\section{GROUP MEMBERS OF THE "COVID-LMIC TASK FORCE"}

Heads: Alfred Papali (Atrium Health, Charlotte, NC, USA) and Marcus Schultz (Mahidol University, Bangkok, Thailand; University of Oxford, Oxford, United Kingdom; Amsterdam University Medical Centers, location "AMC", Amsterdam, The Netherlands).

Advisors: Neill K. J. Adhikari (Interdepartmental Division of Critical Care Medicine, University of Toronto, Toronto, Canada); Emilie J. Calvello Hynes (University of Colorado School of Medicine, Department of Emergency Medicine, Denver, USA); Martin Dünser (Kepler University Hospital and Johannes Kepler University Linz, Linz, Austria) and Mervyn Mer (University of the Witwatersrand, Johannesburg, South Africa).

Subgroup members, in alphabetical order: Andrew Achilleos (Sunnybrook Health Sciences Centre, Toronto, Canada); Hanan Y. Ahmed (Department of Internal Medicine, College of Health Sciences, Addis Ababa University, Addis Ababa, Ethiopia); Kevan M. Akrami (Universidade Federal da Bahia, Faculdade de Medicina, Salvador, Brazil and Division of Infectious Disease, Department of Medicine, University of California San Diego, San Diego, USA); Lia M. Barros (University of Washington, Seattle, USA); B. Jason Brotherton (AIC Kijabe Hospital, Kijabe, Kenya); Sopheakmoniroth Chea (CalmetteHospital, Phnom Penh, Cambodia); William Checkley (The Johns Hopkins University School of Medicine and The Johns Hopkins University Center for Global Health, Baltimore, USA); Elif A. Cizmeci (Interdepartmental Division of Critical Care Medicine, Sunnybrook Health Sciences Centre, University of Toronto, Toronto, Canada); 
Natalie Cobb (University of Washington, Seattle, USA); Finot Debebe (Department of Emergency Medicine and Critical Care, Addis Ababa University School of Medicine, Addis Ababa, Ethiopia); Juliana Ferreira (University of São Paulo, São Paulo, Brasil); Gabriela Galli (University of Pittsburgh School of Medicine, Pittsburgh, USA); Tewodros Haile (Department of Internal Medicine, College of Health Sciences, Addis Ababa University); Bhakti Hansoti (Department of Emergency Medicine, Johns Hopkins University School of Medicine, Baltimore, USA); Madiha Hashmi (Ziauddin University, Karachi, Pakistan); Rebecca Inglis (Lao-Oxford-Mahosot Hospital-Wellcome Trust Research Unit (LOMWRU), Mahosot Hospital, Vientiane, Lao People's Democratic Republic and Department of Intensive Care, Oxford University Hospital Trust, Oxford, United Kingdom); Burton W. Lee (Critical Care Department, NIH, Bethesda, USA; Division of Pulmonary, Allergy and Critical Care, University of Pittsburgh School of Medicine, Pittsburgh, USA); Faith Lelei (AIC Kijabe Hospital, Kijabe, Kenya); Ganbold Lundeg (Mongolian National University of Medical Sciences, Ulan Bator, Mongolia); David Misango (The Aga Khan University, Nairobi, Kenya); Ary Serpa Neto (Department of Critical Care Medicine, Hospital Israelita Albert Einstein, São Paulo, Brazil; Australian and New Zealand Intensive Care Research Centre (ANZIC-RC), Monash University, Melbourne, Australia; Department of Intensive Care, Amsterdam University Medical Centers, location 'AMC', University of Amsterdam, Amsterdam, The Netherlands); Alfred Papali (Atrium Health, Charlotte, NC, USA); Casey Park (Interdepartmental Division of Critical Care Medicine, Sunnybrook Health Sciences Centre, University of Toronto, Toronto, Canada); Rajyabardhan Pattnaik (Ispat General Hospital, Rourkela, India); Jennifer L. Pigoga (Division of Emergency Medicine, University of South Africa, Cape Town, South Africa); Luigi Pisani (Department of Anesthesia and Intensive Care, Miulli Regional Hospital, Acquaviva delle Fonti, Italy; Doctors withAfrica - CUAMM, Padova, Italy; Mahidol-Oxford Tropical Medicine Research Unit (MORU), Mahidol University, Bangkok, Thailand); Elisabeth D. Riviello (Division of Pulmonary, Critical Care, and Sleep Medicine, Beth Israel Deaconess Medical Center and Harvard Medical School, Boston, USA); Kristina E. Rudd (University of Pittsburgh School of Medicine, Pittsburgh, USA); Marcus Schultz (Mahidol University, Bangkok, Thailand; University of Oxford, Oxford, United Kingdom; Amsterdam University Medical Centers, location "AMC", Amsterdam, The Netherlands); Varun U. Shetty (University of Pittsburgh Medical Center, Pittsburgh, USA); Gentle S. Shrestha (Tribhuvan University Teaching Hospital, Kathmandu, Nepal); Chaisith Sivakorn (Mahidol University, Salaya, Thailand); Shaurya Taran (Interdepartmental Division of Critical Care Medicine, University of Toronto, Toronto, Canada); T. Eoin West (University of Washington, Seattle, USA).

Group members of the subgroup "Organization": Natalie Cobb (University of Washington, Seattle, WA); Juliana Ferreira (University of São Paulo, São Paulo, Brazil); Alfred Papali (Atrium Health, Charlotte, NC); Luigi Pisani (Doctors with Africa - CUAMM, Padova, Italy; Mahidol-Oxford Tropical Medicine Research Unit (MORU), Mahidol University, Bangkok, Thailand); and Marcus Schultz (Mahidol University, Bangkok, Thailand; University of Oxford, Oxford, United Kingdom; Amsterdam University Medical Centers, location "AMC," Amsterdam, The Netherlands).

\section{REFERENCES}

1. Wang D et al., 2020. Clinical characteristics of 138 hospitalized patients with 2019 novel coronavirus-infected pneumonia in Wuhan, China. JAMA 323: 1061-1069.

2. Wu Z, McGoogan JM, 2020. Characteristics of and important lessons from the coronavirus disease 2019 (COVID-19) outbreak in China: summary of a report of 72314 cases from the Chinese center for disease control and prevention. JAMA 323: 1239-1242.

3. European Centre for Disease Prevention and Control, 2020. Infection Prevention and Control for COVID-19 in Healthcare Settings-Third Update. Stockholm, Sweden: ECDC.

4. Duy C, Nong VM, Van Ngo A, Doan TT, Nguyen TQ, Truong PT, Olson L, Larsson M, 2020. Nosocomial coronavirus disease outbreak containment, Hanoi, Vietnam, March-April 2020. Emerg Infect Dis 27: 10-17.

5. Li Q et al., 2020. Early transmission dynamics in Wuhan, China, of novel coronavirus-infected pneumonia. $N$ Engl $J$ Med 382: 1199-1207.
6. Rhee C, Baker M, Vaidya V, Tucker R, Resnick A, Morris CA, Klompas M, Program CPE, 2020. Incidence of nosocomial COVID-19 in patients hospitalized at a large US academic medical center. JAMA Netw Open 3: e2020498.

7. Sikkema RS et al., 2020. COVID-19 in health-care workers in three hospitals in the south of The Netherlands: a cross-sectional study. Lancet Infect Dis 20: 1273-1280.

8. Houghton C, Meskell P, Delaney H, Smalle M, Glenton C, Booth A, Chan XHS, Devane D, Biesty LM, 2020. Barriers and facilitators to healthcare workers' adherence with infection prevention and control (IPC) guidelines for respiratory infectious diseases: a rapid qualitative evidence synthesis. Cochrane Database Syst Rev 4: CD013582.

9. Jefferson T et al., 2011. Physical interventions to interrupt or reduce the spread of respiratory viruses. Cochrane Database Syst Rev 2011: CD006207.

10. Krasinski K, LaCouture R, Holzman RS, Waithe E, Bonk S, Hanna $B, 1990$. Screening for respiratory syncytial virus and assignment to a cohort at admission to reduce nosocomial transmission. J Pediatr 116: 894-898.

11. Tan YM et al., 2004. Management of inpatients exposed to an outbreak of severe acute respiratory syndrome (SARS). $J$ Hosp Infect 58: 210-215.

12. Wee LE, Hsieh JYC, Phua GC, Tan Y, Conceicao EP, Wijaya L, Tan $\mathrm{TT}$, Tan BH, 2020. Respiratory surveillance wards as a strategy to reduce nosocomial transmission of COVID-19 through early detection: the experience of a tertiary-care hospital in Singapore. Infect Control Hosp Epidemiol 41: 820-825.

13. Patterson $B$ et al., 2020. A novel cohorting and isolation strategy for suspected COVID-19 cases during a pandemic. J Hosp Infect 105: 632-637.

14. Furstenburg PP, Mukonkole SN, Kibamba CN, Kuiler A, Ngemntu N, Lahri S, Van Hoving DJ, Moodley K, Erasmus E, 2020. Emergency centre reorganization in preparation to the COVID19 pandemic: a district hospital's dynamic adaptation response. Afr J Prim Health Care Fam Med 12: e1-e5.

15. Thomas T, Laher AE, Mahomed A, Stacey S, Motara F, Mer M, 2020. Challenges around COVID-19 at a tertiary-level healthcare facility in South Africa and strategies implemented for improvement. S Afr Med J 110: 964-967.

16. CDC, 2020. Interim Infection Prevention and Control Recommendations for Patients with Suspected or Confirmed Coronavirus Disease 2019 (COVID-19) in Healthcare Settings. Available at: https://www.cdc.gov/coronavirus/2019-ncov/ hcp/infection-control-recommendations.html. Accessed June 29, 2020.

17. Islam MS, Rahman KM, Sun Y, Qureshi MO, Abdi I, Chughtai AA, Seale $H, 2020$. Current knowledge of COVID-19 and infection prevention and control strategies in healthcare settings: a global analysis. Infect Control Hosp Epidemiol 41: 1196-1206.

18. World Health Organization, 2020. Infection Prevention and Control during Health Care when COVID-19 Is Suspected: Interim Guidance. Geneva, Switzerland: WHO.

19. World Health Organization, 2020. Clinical Management of COVID19: Interim Guidance. Geneva, Switzerland: WHO.

20. World Health Organization, 2020. Severe Acute Respiratory Infections Treatment Centre: Practical Manual to Set up and Manage a SARI Treatment Centre and SARI Screening Facility in Health Care Facilities. Geneva, Switzerland: WHO.

21. Siow WT, Liew MF, Shrestha BR, Muchtar F, See KC, 2020. Managing COVID-19 in resource-limited settings: critical care considerations. Crit Care 24: 167.

22. Africa CDC, 2020. Guidance on Setting Up an Isolation Ward for COVID-19 Cases Addis Ababa, Ethiopia: Africa CDC and African Union.

23. Inglis R, Barros L, Checkley W, Cizmeci EA, Lelei-Mailu F, Pattnaik R, Papali A, Schultz MJ, Ferreira JC, 2020. Pragmatic recommendations for safety while caring for hospitalized patients with coronavirus disease 2019 (COVID-19) in low-and middle-income countries. Am J Trop Med Hyg doi: 10.4269/ajtmh.20-1128.

24. Africa CDC, 2020. COVID-19 Infection Prevention and Control: Your Questions Answered Addis Ababa, Ethiopia: Africa CDC and African Union. 
25. Oran DP, Topol EJ, 2020. Prevalence of asymptomatic SARSCoV-2 infection: a narrative review. Ann Intern Med 173: 362-367.

26. Nguyen LH et al., 2020. Risk of COVID-19 among front-line health-care workers and the general community: a prospective cohort study. Lancet Public Health 5: e475-e483.

27. Lai J et al., 2020. Factors associated with mental health outcomes among health care workers exposed to coronavirus disease 2019. JAMA Netw Open 3: e203976.

28. Sahu AK, Amrithanand VT, Mathew R, Aggarwal P, Nayer J, Bhoi S, 2020. COVID-19 in health care workers - a systematic review and meta-analysis. Am J Emerg Med 38: 1727-1731.

29. Rickman HM, Rampling T, Shaw K, Martinez-Garcia G, Hail L, Coen P, Shahmanesh M, Shin GY, Nastouli E, Houlihan CF, 2020. Nosocomial transmission of coronavirus disease 2019: a retrospective study of 66 hospital-acquired cases in a London teaching hospital. Clin Infect Dis ciaa816.

30. Vandyck-Sey P, Amoh G, Essuman A, Lawson H, 2020. Incidental finding of COVID-19 infection amongst staff at a primary care facility in Ghana. Afr J Prim Health Care Fam Med 12: e1-e4.

31. Esswein EJ, Kiefer M, Wallingford K, Burr G, Lee LJ, Wang JD, Wang SC, Su IJ, 2004. Environmental and occupational health response to SARS, Taiwan, 2003. Emerg Infect Dis 10: 1187-1194.

32. McDonald LC, Simor AE, Su IJ, Maloney S, Ofner M, Chen KT, Lando JF, McGeer A, Lee ML, Jernigan DB, 2004. SARS in healthcare facilities, Toronto and Taiwan. Emerg Infect Dis 10: 777-781.

33. Dwosh HA, Hong HH, Austgarden D, Herman S, Schabas R, 2003. Identification and containment of an outbreak of SARS in a community hospital. CMAJ 168: 1415-1420.

34. Svoboda T et al., 2004. Public health measures to control the spread of the severe acute respiratory syndrome during the outbreak in Toronto. N Engl J Med 350: 2352-2361.

35. Fischer WA, II, Hynes NA, Perl TM, 2014. Protecting health care workers from Ebola: personal protective equipment is critical but is not enough. Ann Intern Med 161: 753-754.

36. Olu O et al., 2015. Epidemiology of Ebola virus disease transmission among health care workers in Sierra Leone, May to December 2014: a retrospective descriptive study. BMC Infect Dis 15: 416.

37. European CDC, 2020. COVID-19 Clusters and Outbreaks in Occupational Settings in the EU/EEA and the UK. Stockholm, Sweden: ECDC.

38. Chersich MF et al., 2020. COVID-19 in Africa: care and protection for frontline healthcare workers. Glob Health 16: 46.

39. Logie $\mathrm{CH}$, Turan JM, 2020. How do we balance tensions between COVID-19 public health responses and stigma mitigation? Learning from HIV research. AIDS Behav 24: 2003-2006.

40. Ran L, Chen X, Wang Y, Wu W, Zhang L, Tan X, 2020. Risk factors of healthcare workers with corona virus disease 2019: a retrospective cohort study in a designated hospital of Wuhan in China. Clin Infect Dis 71: 2218-2221.

41. Rothe $\mathrm{C}$ et al., 2020. Transmission of 2019-nCoV infection from an asymptomatic contact in Germany. N Engl J Med 382: 970-971.

42. Huang L, Zhang X, Zhang X, Wei Z, Zhang L, Xu J, Liang P, Xu Y, Zhang C, Xu A, 2020. Rapid asymptomatic transmission of COVID-19 during the incubation period demonstrating strong infectivity in a cluster of youngsters aged 16-23 years outside Wuhan and characteristics of young patients with COVID-19: a prospective contact-tracing study. J Infect 80: e1-e13.

43. Böhmer MM et al., 2020. Investigation of a COVID-19 outbreak in Germany resulting from a single travel-associated primary case: a case series. Lancet Infect Dis 20: 920-928.

44. Mukhopadhyay A, Tambyah PA, Singh KS, Lim TK, Lee KH, 2004. SARS in a hospital visitor and her intensivist. J Hosp Infect 56: 249-250.

45. Ki HK, Han SK, Son JS, Park SO, 2019. Risk of transmission via medical employees and importance of routine infectionprevention policy in a nosocomial outbreak of Middle East respiratory syndrome (MERS): a descriptive analysis from a tertiary care hospital in South Korea. BMC Pulm Med 19: 190.

46. Hui DS, Azhar El, Kim YJ, Memish ZA, Oh MD, Zumla A, 2018. Middle East respiratory syndrome coronavirus: risk factors and determinants of primary, household, and nosocomial transmission. Lancet Infect Dis 18: e217-e227.

47. Kim KH, Tandi TE, Choi JW, Moon JM, Kim MS, 2017. Middle east respiratory syndrome coronavirus (MERS-CoV) outbreak in South Korea, 2015: epidemiology, characteristics and public health implications. J Hosp Infect 95: 207-213.

48. Cho SY et al., 2016. MERS-CoV outbreak following a single patient exposure in an emergency room in South Korea: an epidemiological outbreak study. Lancet 388: 994-1001.

49. Hastings DL et al., 2016. Outbreak of middle east respiratory syndrome at tertiary care hospital, Jeddah, Saudi Arabia, 2014. Emerg Infect Dis 22: 794-801.

50. Ofner-Agostini M, Wallington T, Henry B, Low D, McDonald LC, Berger L, Mederski B, Wong T, 2008. Investigation of the second wave (phase 2) of severe acute respiratory syndrome (SARS) in Toronto, Canada. What happened? Can Commun Dis Rep 34: 1-11.

51. CDC, 2003. Update: severe acute respiratory syndrome--Toronto, Canada, 2003. MMWR Morb Mortal Wkly Rep 52: 547-550.

52. Gopalakrishna G, Choo P, Leo YS, Tay BK, Lim YT, Khan AS, Tan CC, 2004. SARS transmission and hospital containment. Emerg Infect Dis 10: 395-400.

53. Ho PL, Tang XP, Seto WH, 2003. SARS: hospital infection control and admission strategies. Respirology 8 (Suppl 1): S41-S45.

54. Chow KY, Lee CE, Ling ML, Heng DM, Yap SG, 2004. Outbreak of severe acute respiratory syndrome in a tertiary hospital in Singapore, linked to an index patient with atypical presentation: epidemiological study. BMJ 328: 195.

55. Varia M, Wilson S, Sarwal S, McGeer A, Gournis E, Galanis E, Henry B, 2003. Investigation of a nosocomial outbreak of severe acute respiratory syndrome (SARS) in Toronto, Canada. CMAJ 169: 285-292.

56. Shen Z, Ning F, Zhou W, He X, Lin C, Chin DP, Zhu Z, Schuchat A, 2004. Superspreading SARS events, Beijing, 2003. Emerg Infect Dis 10: 256-260.

57. Hua $\mathrm{J}$ et al., 2020. Epidemiological features and medical careseeking process of patients with COVID-19 in Wuhan, China. ERJ Open Res 6: 00142-2020.

58. Valley TS, Schutz A, Nagle MT, Miles LJ, Lipman K, Ketcham SW, Kent M, Hibbard CE, Harlan EA, Hauschildt K, 2020. Changes to visitation policies and communication practices in Michigan ICUs during the COVID-19 pandemic. Am J Respir Crit Care Med 202: 883-885.

59. Foster S, 2020. Open visiting in the NHS. Br J Nurs 29: 491.

60. Liu YA, Hsu YC, Lin MH, Chang HT, Chen TJ, Chou LF, Hwang SJ, 2020. Hospital visiting policies in the time of coronavirus disease 2019: a nationwide website survey in Taiwan. J Chin Med Assoc 83: 566-570.

61. Hsu YC, Liu YA, Lin MH, Lee HW, Chen TJ, Chou LF, Hwang SJ, 2020. Visiting policies of hospice wards during the COVID-19 pandemic: an environmental scan in Taiwan. Int J Environ Res Public Health 17: 2857.

62. McMichael TM et al., 2020. COVID-19 in a long-term care facility King county, Washington, February 27-March 9, 2020. MMWR Morb Mortal Wkly Rep 69: 339-342.

63. Munoz-Price LS, Banach DB, Bearman G, Gould JM, Leekha S, Morgan DJ, Palmore TN, Rupp ME, Weber DJ, Wiemken TL, 2015. Isolation precautions for visitors. Infect Control Hosp Epidemiol 36: 747-758.

64. Weber DJ, Rutala WA, Fischer WA, Kanamori H, Sickbert-Bennett EE, 2016. Emerging infectious diseases: focus on infection control issues for novel coronaviruses (severe acute respiratory syndrome-CoV and middle east respiratory syndrome-CoV), hemorrhagic fever viruses (Lassa and Ebola), and highly pathogenic avian influenza viruses, $\mathrm{A}(\mathrm{H} 5 \mathrm{~N} 1)$ and $\mathrm{A}(\mathrm{H} 7 \mathrm{~N} 9)$. $A m \mathrm{~J}$ Infect Control 44: e91-e100.

65. Adegboye OA, Elfaki F, 2018. Network analysis of MERS coronavirus within households, communities, and hospitals to identify most centralized and super-spreading in the Arabian peninsula, 2012 to 2016. Can J Infect Dis Med Microbiol 2018: 6725284.

66. Al-Dorzi HM, Aldawood AS, Khan R, Baharoon S, Alchin JD, Matroud AA, Al Johany SM, Balkhy HH, Arabi YM, 2016. The critical care response to a hospital outbreak of middle east 
respiratory syndrome coronavirus (MERS-CoV) infection: an observational study. Ann Intensive Care 6: 101.

67. James L, Shindo N, Cutter J, Ma S, Chew SK, 2006. Public health measures implemented during the SARS outbreak in Singapore, 2003. Public Health 120: 20-26.

68. Shaw K, 2006. The 2003 SARS outbreak and its impact on infection control practices. Public Health 120: 8-14.

69. Chan KP, 2005. Control of severe acute respiratory syndrome in Singapore. Environ Health Prev Med 10: 255-259.

70. SARS Investigation Team from DMERI, SGH, 2005. Strategies adopted and lessons learnt during the severe acute respiratory syndrome crisis in Singapore. Rev Med Virol 15: 57-70.

71. Twu SJ et al., 2003. Control measures for severe acute respiratory syndrome (SARS) in Taiwan. Emerg Infect Dis 9: 718-720.

72. Lee NE, Siriarayapon P, Tappero J, Chen KT, Shuey D, Limpakarnjanarat K, Chavavanich A, Dowell SF, 2004. Infection control practices for SARS in Lao People's Democratic Republic, Taiwan, and Thailand: experience from mobile SARS containment teams, 2003. Am J Infect Control 32: 377-383.

73. Buchbinder N, Dumesnil C, Pinquier D, Merle V, Filhon B, Schneider P, Vannier JP, 2011. Pandemic A/H1N1/2009 influenza in a paediatric haematology and oncology unit: successful management of a sudden outbreak. $J$ Hosp Infect 79: 155-160.

74. Chaovavanich A et al., 2004. Early containment of severe acute respiratory syndrome (SARS); experience from Bamrasnaradura Institute, Thailand. J Med Assoc Thai 87: 1182-1187.

75. Beck M, Antle BJ, Berlin D, Granger M, Meighan K, Neilson BJ, Shama W, Westland J, Kaufman M, 2004. Wearing masks in a pediatric hospital: developing practical guidelines. Can J Public Health 95: 256-257.

76. Leung TF et al., 2004. Infection control for SARS in a tertiary paediatric centre in Hong Kong. J Hosp Infect 56: 215-222.

77. CDC, 2020. Management of Visitors to Healthcare Facilities in the Context of COVID-19: Non-US Healthcare Settings. Available at: https://www.cdc.gov/coronavirus/2019-ncov/hcp/non-ussettings/hcf-visitors.html. Accessed June 29, 2020.

78. Africa CDC, 2020. Standard Operating Procedures for Caregivers in COVID-19 Treatment Centres Addis Ababa, Ethiopia: Africa $\mathrm{CDC}$ and African Union.

79. Powell-Jackson T, King JJC, Makungu C, Spieker N, Woodd S, Risha P, Goodman C, 2020. Infection prevention and control compliance in Tanzanian outpatient facilities: a cross-sectional study with implications for the control of COVID-19. Lancet Glob Health 8: e780-e789.

80. Rogers S, 2004. Why can't I visit? The ethics of visitation restrictions - lessons learned from SARS. Crit Care 8: 300-302.

81. Bakar M, Capano E, Patterson M, McIntyre B, Walsh CJ, 2020. The role of palliative care in caring for the families of patients with COVID-19. Am J Hosp Palliat Med 37: 866-868.

82. Carlucci M, Carpagnano LF, Dalfino L, Grasso S, Migliore G, 2020. Stand by me 2.0. Visits by family members at COVID-19 time. Acta Biomed 91: 71-74.

83. Moolla MS, Broadhurst A, Parker MA, Parker A, Mowlana A, 2020. Implementing a video call visit system in a coronavirus disease 2019 unit. Afr J Prim Health Care Fam Med 12: e1-e3.

84. Chung JW et al., 2004. Rapid assessment of a helpdesk service supporting severe acute respiratory syndrome patients and their relatives. J Clin Nurs 13: 748-755.

\section{APPENDIX}

Development of recommendations and suggestions. Selection of Task Force members. The selection of the group members was based on interest in specific aspects of novel coronavirus disease (COVID-19) and direct experience in lowand middle-income countries (LMICs). Alfred Papali and Marcus Schultz contacted potential team members through email and in person early in the pandemic of COVID-19, and created subgroups assigned to separate areas in COVID-19 management, i.e., "triage," "safety," "organization," "diagnostics and prognostic modeling," "acute respiratory failure,"
TABLE A1

Quality of Evidence

\begin{tabular}{lll}
\hline A & Randomized clinical trials & High \\
B & $\begin{array}{c}\text { Downgraded randomized clinical trial(s) or } \\
\text { upgraded observational studies }\end{array}$ & Moderate \\
C & $\begin{array}{l}\text { Observational studies } \\
\text { Downgraded observational studies or } \\
\text { expert opinions }\end{array}$ & Low \\
& $\begin{array}{l}\text { Very Low } \\
\text { F }\end{array}$ &
\end{tabular}

Factors that may decrease strength of evidence include high likelihood of bias; inconsistency of results, including problems with subgroup analyses; indirectness of ince (other population, intervention, control, outcomes, comparison); imprecision of findings; and likelihood of reporting bias.

Factors that may increase strength of evidence: large magnitude of effect (direct evidence, relative risk $>2$ with no plausible confounders); very large magnitude of effect with relative risk $>5$ and no threats to validity (by two levels); and dose-response gradient.

Adapted from Dondorp AM, Dünser MW, Schultz MJ, eds., 2019. Sepsis Management in Resource-limited Settings. Springer. doi.org/10.1007/978-3-030-03143-5

"acute kidney injury," "coagulopathy," "prevention and therapy," "shock," "therapeutics," and "support after initial care." In total, there were 38 Task Force members representing five medical specialties or disciplines (emergency medicine, intensive care, infectious diseases, internal medicine and critical care nursing) from five out of six World Health Organization (WHO) geographic regions. The Task Force consisted of 16 fulltime LMIC members, 16 full time high-income country (HIC) members-all with direct LMIC experience-and 6 members with joint LMIC/HIC appointments.

Selection of subgroup members. Natalie Cobb, Marcus Schultz, Alfred Papali, and Juliana Ferreira were assigned to this subgroup based on their specific expertise and interest in the topic.

Discussions. The subgroup worked via electronic-based communications to establish the procedures for the literature review and drafting of tables for evidence analysis. Discussions occurred both within the subgroup and with members of other subgroups. First, a set of clearly defined questions regarding the organization of healthcare facilities for infection

TABLE A2

Strong versus Weak Recommendations ${ }^{\star}$

\begin{tabular}{|c|c|}
\hline What is Considered & How it affects the recommendation \\
\hline High evidence & $\begin{array}{l}\text { The higher the quality of evidence, } \\
\text { the more likely a strong } \\
\text { recommendation. }\end{array}$ \\
\hline $\begin{array}{l}\text { Certainty about the balance of } \\
\text { benefits vs. harms and burdens }\end{array}$ & $\begin{array}{l}\text { The larger/smaller the difference } \\
\text { between the desirable and } \\
\text { undesirable consequences and } \\
\text { the certainty around that } \\
\text { difference, the more likely a } \\
\text { strong/weak recommendation. }\end{array}$ \\
\hline Certainty in or similar values & $\begin{array}{l}\text { The more certainty or similarity in } \\
\text { values and preferences, the } \\
\text { more likely a strong } \\
\text { recommendation. }\end{array}$ \\
\hline Resource implications & $\begin{array}{l}\text { The lower/higher the cost of an } \\
\text { intervention compared to the } \\
\text { alternative the more likely a } \\
\text { strong/weak recommendation. }\end{array}$ \\
\hline $\begin{array}{l}\text { Availability and feasibility in } \\
\text { LMICs }\end{array}$ & $\begin{array}{l}\text { The less available, the more likely } \\
\text { a weak recommendation. }\end{array}$ \\
\hline Affordability for LMICs & $\begin{array}{l}\text { The less affordable, the more } \\
\text { likely a weak recommendation. }\end{array}$ \\
\hline Safety of the intervention in LMICs & $\begin{array}{l}\text { The less safe in an LMIC, the more } \\
\text { likely a weak recommendation. }\end{array}$ \\
\hline
\end{tabular}

*In case of a strong recommendation we use "we recommend ..."; in case of a weak recommendation we use "we suggest ..."

Adapted from Dondorp AM, Dünser MW, Schultz MJ, eds., 2019. Sepsis Management in Resource-limited Settings. Springer. doi.org/10.1007/978-3-030-03143-5 
prevention and control were formulated. These were reviewed for content and clarity by the subgroup members and heads from the other subgroups. After approval by the subgroup members and heads from the other subgroups, the subgroup members split up, each seeking evidence for recommendations regarding the specific questions posed, seeking help from the subgroup members in identifying relevant publications where necessary. During this process, questions could be combined, so the subgroup was finally left with three major questions. The subgroup summarized the evidence in a report and formulated a set of recommendations and suggestions after several online discussions. After approval within the subgroup, the report was sent for approval by all members of the Task Force in two rounds.

Search techniques. The literature search followed the same techniques as previously described. Searches were conducted in PubMed and the Cochrane Libraries. Furthermore, the subgroup members identified investigations from LMICs and also searched for unpublished study results. The initial literature search was conducted through May 30,2020. During the revisions process of the manuscript, an updated search was conducted through November 1, 2020. Lastly, we reviewed guidelines from the World Health Organization, Africa Centres for Disease Control and Prevention, United States Centers for Disease Control and Prevention, and the European Centre for Disease Prevention and Control.
Grading of Recommendations. The subgroup members classified quality of evidence as high, moderate, low, or very low and recommendations as strong or weak. The factors influencing this classification are presented in Appendix Table A1.

The subgroup members paid extensive attention to availability, feasibility, and safety matters in LMICs. A strong recommendation was worded as "we recommend" and a weak recommendation as "we suggest". A number of recommendations could remain "ungraded" (UG), when, in the opinion of the subgroup members, such recommendations were not conducive for the process described above (Appendix Table A2).

Reporting. The report was edited for style and form by Alfred Papali or Marcus Schultz, with final approval by the subgroup and then by the entire "COVID-LMIC Task Force." A final document was submitted to the "American Journal of Tropical Medicine and Hygiene" for potential publication and made open access.

Conflicts of interest. No members of the 'organization' subgroup represented industry, and there was no industry input into guidelines development. No member of the 'organization' subgroup received honoraria for any role in the guideline development process. None reported conflicts of interest. Open access fees for this manuscript, and all 9 others in the series, were supported by the Wellcome Trust of Great Britain. 